Short Communication

Evolutionary Genetics

\title{
Tracking a recent horizontal transfer event: The $P$-element reaches Brazilian populations of Drosophila simulans
}

\author{
Ana M.L. Nascimento ${ }^{1}$, Bráulio S.M.L. Silva ${ }^{1} \mathbb{0}$, Marta Svartman $^{1}$ and Gustavo C.S. Kuhn ${ }^{1}$ \\ ${ }^{1}$ Universidade Federal de Minas Gerais, Departamento de Genética, Ecologia e Evolução, Belo Horizonte, \\ $M G$, Brazil.
}

\begin{abstract}
The "cut-and-paste" P-element present in some Diptera illustrates two important transposable elements abilities: to move within genomes and to be transmitted between non-mating species, a phenomenon known as horizontal transposon transfer (HTT). Recent studies reported a HTT of the P-element from Drosophila melanogaster to $D$. simulans. P-elements first appeared in D. simulans European samples collected in 2006 and spread across several populations from Europe, Africa, North America and Japan within seven years. Nevertheless, no $P$-element was found in South American populations of D. simulans collected between 2002 and 2009. We investigated the presence of the $P$-element in $D$. simulans collected in five Brazilian localities between 2018 and 2019, using a combination of methodologies such as PCR, DNA sequencing and FISH on chromosomes. Our experiments revealed the presence of the $P$-element in all sampled individuals from the five localities. The number of $P$-elements per individual varied from 11 to 20 copies and truncated copies were also observed. Altogether, our results showed that $P$-element invasion in $D$. simulans is at an advanced stage in Brazil and, together with other recent studies, confirms the remarkable rapid invasion of $P$-elements across worldwide $D$. simulans populations.
\end{abstract}

Keywords: Horizontal transfer, P-element, transposable elements, Drosophila simulans.

Received: October 21, 2019; Accepted: March 18, 2020.

Transposable elements (TEs) are DNA sequences usually less than $15 \mathrm{~kb}$ long that possess an intrinsic ability to mobilize and change their genomic location, with new copies generated during the process. They are abundant components of eukaryote genomes and play an important role in generating genetic variation that impacts the evolution of the species in which they transit (Kidwell and Lisch, 2001; Schaack et al., 2010; Bourque et al., 2018).

The $P$-element is a well-studied eukaryotic transposable element in Diptera (reviewed by Rio, 1991; Clark et al., 1994; Castro and Carareto, 2004). It is a "cut-andpaste" DNA transposon that excises as double strand DNA and reinserts elsewhere in the genome. Full autonomous elements are approximately 2,900 bp long but several copies may contain deletions and therefore be either non-autonomous or completely inactive. The $P$-element illustrates two important features of transposable elements: their ability to jump within genomes and their capacity of spreading between non-mating species, a phenomenon known as horizontal transposon transfer (HTT) (Silva and Kidwell, 2000).

Send correspondence to Gustavo C.S. Kuhn. Universidade Federal de Minas Gerais Departamento de Genética, Ecologia e Evolução, Av. Antônio Carlos 6627, Postal Code: 31270-901, Pampulha, Belo Horizonte, MG, Brazil. E-mail: gcskuhn@ufmg.br
When TEs invade a new species, they are rapidly amplified and cause a broad spectrum of effects (reviewed by Schaack et al., 2010). At a later stage, the transposition activity is regulated or suppressed by several mechanisms that lead to a decrease in transposition rate, accumulation of mutations, excision and eventually extinction, unless a HTT event introduces the TE in a new species, leading to its persistence over time (Loreto et al., 2008; Schaack et al., 2010). Therefore, HTT is considered an important event within the life cycle of TEs and consequently for the evolution of genomes and species.

The first reported case of HTT in Drosophila involved the transfer of the P-element from Drosophila willistoni to $D$. melanogaster, an event that probably took place in the American continent around the 1950s (Anxolabéhère et al., 1988). After this invasion, $P$-elements rapidly amplified intra-genomically and spread through $D$. melanogaster worldwide natural populations within five decades (Anxolabéhère et al., 1988). As a further evidence of a recent HTT, the $P$-element from $D$. willistoni is nearly identical (only a single nucleotide difference) to that of $D$. melanogaster.

Remarkably, a second invasion has been recently reported, this time involving a $D$. melanogaster $P$-element variant into Drosophila simulans, in which no $P$-elements had previously been detected (Kofler et al., 2015). D. mela- 
nogaster and $D$. simulans are closely related species from the melanogaster subgroup and are separated by approximately $\sim 5.4$ Mya from their common ancestor (Tamura et al., 2004). Both species share common ecological habits, similar geographic distribution and insertions from different TE families (Sánchez-Gracia et al., 2005; Bartolomé et $a l ., 2009)$. The $P$-element that invaded $D$. simulans differs from the most common $D$. melanogaster variant by a nucleotide substitution at position $2040(\mathrm{G} \rightarrow \mathrm{A})$ in intron 3. This substitution is also found in D. melanogaster, but at very low frequencies (0.16-2\%) (Kofler et al., 2015; Yoshitake et al., 2018).

This second $P$-element invasion is supposed to be recent, since it was first detected in $D$. simulans collected in Europe only from 2006 on. In the following seven years, it rapidly spread across several $D$. simulans populations from Europe, Africa, North America and Japan (Hill et al., 2016; Yoshitake et al., 2018). The exact mechanism responsible for this HTT has not been discovered yet. In South America, the presence of $P$-elements has been previously investigated in D. simulans from a small sample of flies collected between 2002 and 2009 from one locality each in Brazil $($ Ubatuba $=9$ flies $)$, Peru $($ Cusco $=1$ fly) and Colombia (Guaymaral $=2$ flies) (Hill et al., 2016). Although none of these localities showed flies with $P$-elements, it cannot be ruled out the possibility that $P$-elements were not detected due to the limited sampling.

In order to investigate whether the $P$-element reached South American D. simulans populations, we looked for its presence in flies recently collected in Brazil. Collection trips using conventional banana bait traps were made between June 2018 and January 2019 in five Brazilian localities: Ubatuba (SP01), São Carlos (SP02), Sorocaba (SP03), Serra do Cipó (MG01) and Caldas Novas (GO01) (Figure 1 and Table 1). D. simulans identification was based on morphological characters and confirmed by PCR using the primers (cidsimF 5' GGTATTATTTGCTTTGCTTG 3' and cidsimR 5' CTGAGCTACCATTCGTTGG 3') that we designed to specifically amplify a fragment from the Cid gene (Centromere Identifier) of D. simulans (Figure S1).

We tested the presence of the $P$-element in three to six individuals from each locality. PCR was performed with oligos that amplify $P$-element exons $0,1,2$, and 3 , as described by Hill et al. (2016). Amplicons corresponding to all four $P$-element exons were visualized in all sampled individuals (Figure S2). In order to further confirm the $\mathrm{G} \rightarrow$ A substitution present in $D$. simulans $P$-element copies, we PCR-amplified and sequenced a pool of non-cloned intron 3 sequences from individuals representing all five localities (see Internet Resources Section for chromatogram files). A sequence alignment containing the intron 3 sequences from our D. simulans samples together with a reference P-element from D. melanogaster (Flybase ID: FBte0000037) showed that all $D$. simulans $P$-elements share the nucleotide A at position 2040 (Figure 2), which is considered as a marker of this $P$-element invasion in $D$. simulans (Kofler et al., 2015; Yoshitake et al., 2018). In summary, the PCR and sequencing experiments showed that the $P$-element is pres-

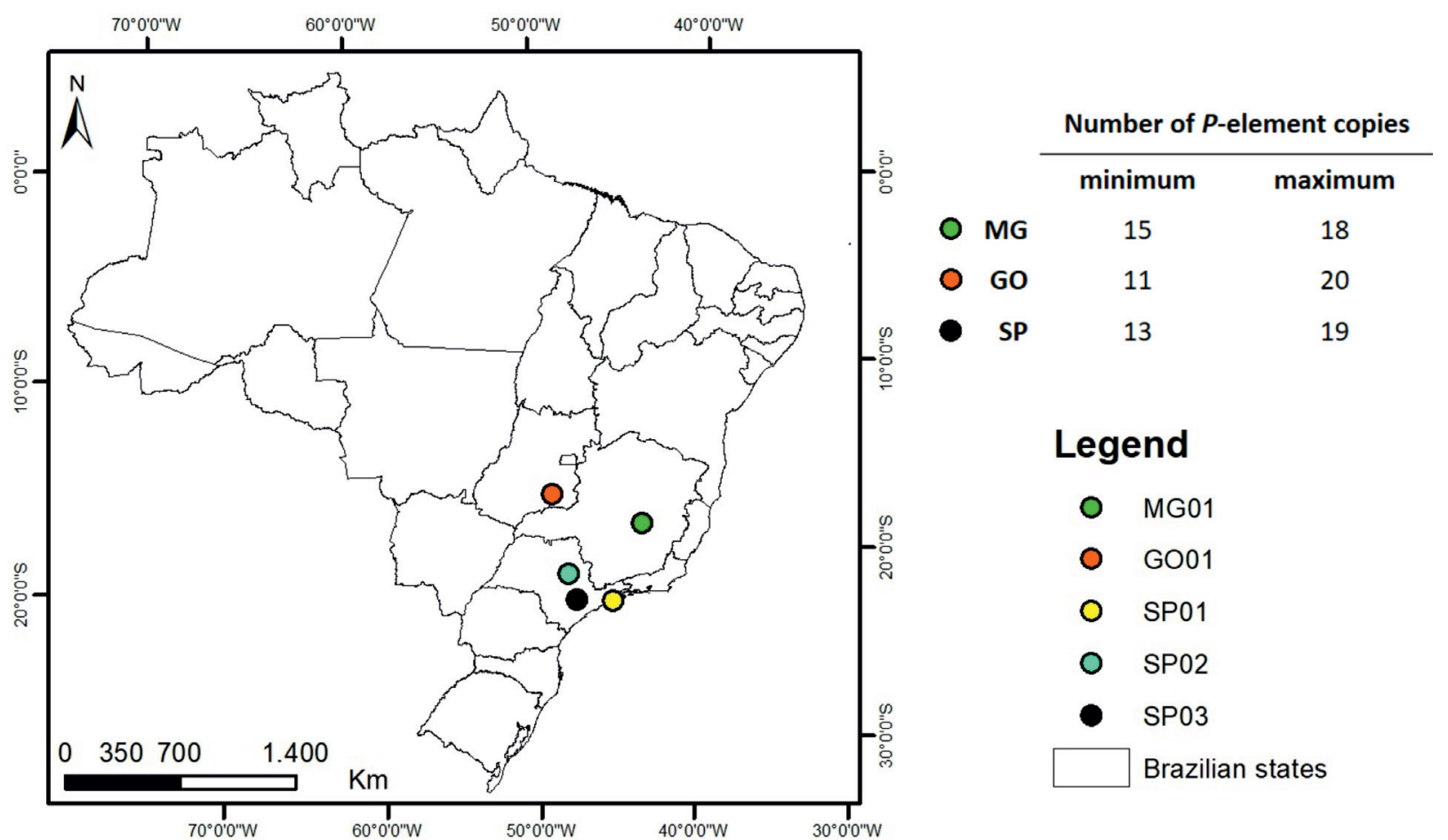

Figure 1 - Approximate geographic locations of the Brazilian $D$. simulans populations studied. The table on the right shows the number of $P$-element copies estimated for three locations studied. 
Table 1 - Brazilian Drosophila simulans populations analysed for P-element presence.

\begin{tabular}{|c|c|c|c|}
\hline Number of analysed specimens & Location and code & Coordinates & Date \\
\hline 6 & Serra do Cipó, MG (MG01) & $19^{\circ} 17^{\prime} 29.9^{\prime \prime} \mathrm{S} 43^{\circ} 33^{\prime} 34.9^{\prime \prime} \mathrm{W}$ & $06 / 18 / 2018$ \\
\hline 6 & Caldas Novas, GO (GO01) & $17^{\circ} 39^{\prime} 40.6^{\prime \prime} \mathrm{S} 48^{\circ} 44^{\prime} 30.6^{\prime \prime} \mathrm{W}$ & $07 / 25 / 2018$ \\
\hline 3 & Ubatuba, SP (SP01) & $23^{\circ} 32^{\prime} 35.9^{\prime \prime} \mathrm{S} 45^{\circ} 13^{\prime} 56.1$ '’ W & $01 / 28 / 2019$ \\
\hline 6 & São Carlos, SP (SP02) & $22^{\circ} 00^{\prime} 53.9^{\prime \prime} \mathrm{S} 47^{\circ} 54^{\prime} 03.8^{\prime \prime} \mathrm{W}$ & $07 / 26 / 2018$ \\
\hline 4 & Sorocaba, SP (SP03) & $23^{\circ} 29^{\prime} 26.3^{\prime \prime S} 47^{\circ} 25^{\prime} 24.1^{\prime \prime} \mathrm{W}$ & $01 / 28 / 2019$ \\
\hline
\end{tabular}

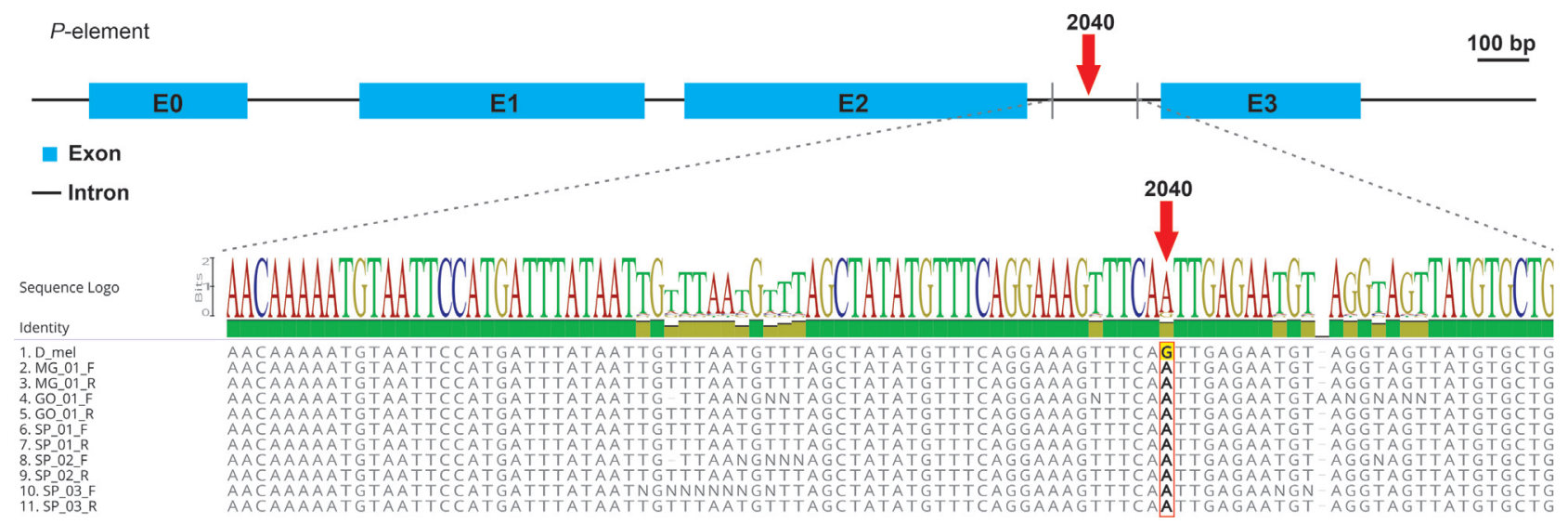

Figure 2 - Schematic representation of a full $P$-element sequence (above). Nucleotide alignment containing partial intron 3 sequences from the five $D$. simulans sampled populations together with the reference sequence (Flybase ID: FBte0000037) from D. melanogaster (D_mel) (below). Position 2040 (highlighted) has the nucleotide A in all $D$. simulans sequences, which is a marker of this species' $P$-element. DNA sequencing was performed with Forward (F) and Reverse (R) primers directly from non-cloned PCR products. 'N' denotes nucleotide positions with low sequencing quality in the chromatograms.

ent in all D. simulans sampled individuals from the five studied Brazilian localities.

In order to access the abundance of $P$-elements in $D$. simulans from Brazil, we performed double-FISH on polytene chromosomes, using as probes a $551 \mathrm{bp}$ segment of exon 1 and a 662 bp fragment from exon 2. FISH experiments were conducted as described in Dias et al. (2014). A total of 15 larvae from three isofemale lines (DS-MG01, DS-GO01, DS-SP03) representing three localities (Serra do Cipó, Caldas Novas and Sorocaba) were analysed. The FISH experiments revealed $P$-element copies distributed along several euchromatic loci (see Figure 3). Despite the fact that previous studies suggested a preferential insertion of $P$-elements at subtelomeric regions (Karpen and Spradling, 1992; Kofler et al., 2018), we detected only two signals at this location (Figure 3). The two probes co-hybridized except in a few cases in which only one probe hybridized indicating the existence of divergent or truncated $P$-element copies (Figure 3 ). Because the $P$-element invaded $D$. simulans very recently and in a single horizontal transfer event, all copies are very homogeneous (Kofler et $a l ., 2015)$. On the other hand, $P$-elements with internal deletions were reported in $D$. simulans experimental populations after 20 generations following their genomic spread from a single copy (Kofler et al., 2018), while in natural populations $P$-elements with at least one missing exon were reported (Hill et al., 2016). The presence of truncated copies is not uncommon, but their detection in our $D$. simulans samples is important because they can repress the mobilization of $P$-elements through the expression of nonfunctional transposases that compete with functional ones for binding sites (Lee et al., 1998; Kofler et al., 2018).

Based on the signals revealed by our FISH experiments, the number of $P$-element copies in three Brazilian sampled populations varied from 11 to 20 copies per individual. However, these numbers should be taken as underestimates, because the same hybridization signal may contain more than one copy or some copies may lack the two exons segments used as probes.

We also investigated the $P$-element presence in additional species captured with $D$. simulans at the same bait, but without previous record of $P$-elements in their genomes. The PCR using oligos for $P$-element exons $0,1,2$, and 3 produced no amplicons in all tested species: Zaprionus indianus, D. nasuta, D. malerkotliana, D. mediopicta, D. ananassae, D. mirim and one species from the Cardini group, all collected in 2019 (Figure S3). Whereas this result is insufficient to draw general conclusions, it may be useful as a reference for future studies tracking $P$-element invasions in other Drosophila species. In fact, Serrato-Capuchina et al. (2018) reported that $P$-elements also recently 


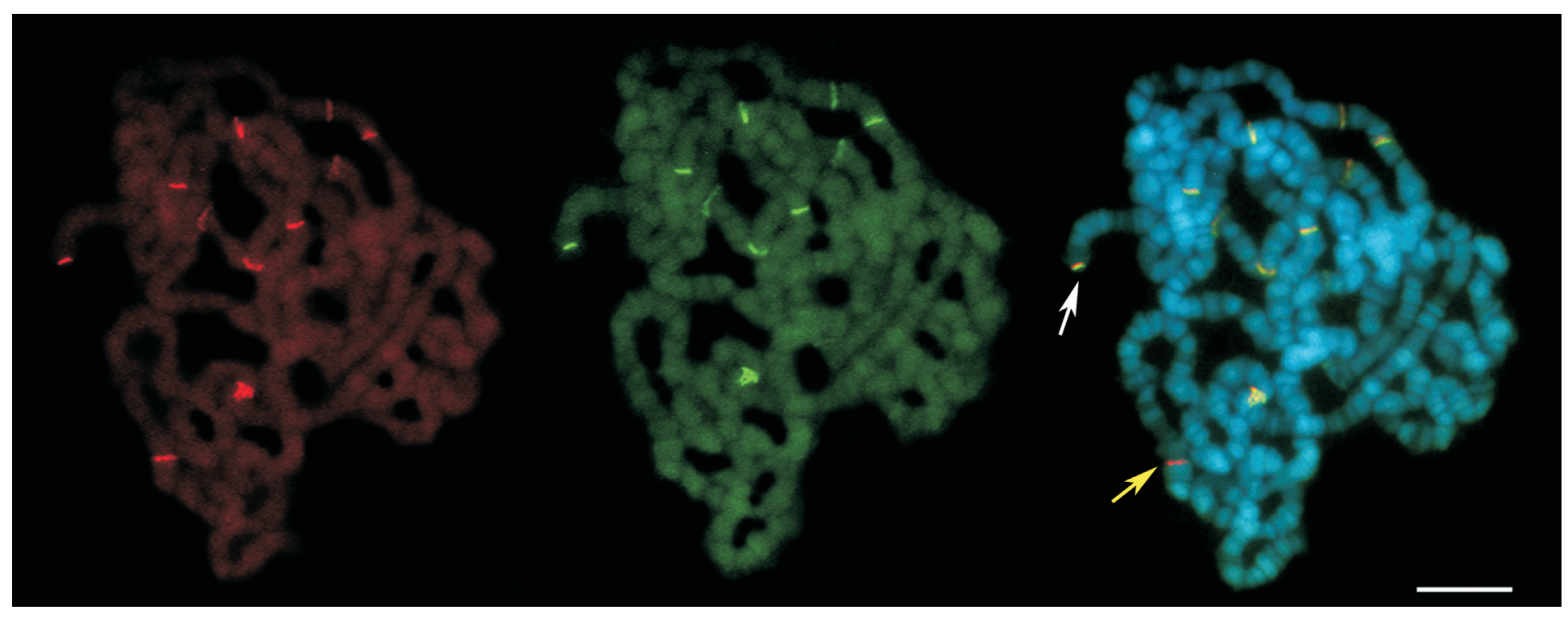

Figure 3 - FISH on polytene chromosome of $D$. simulans (locality GO01) using two $P$-element probes: exon 1-red (left panel), exon 2-green (middle panel), and exon $1+2$ (right panel). The chromosome was counterstained with DAPI-blue. Both arrows point to terminal regions insertions. The white arrow indicates co-hybridization of exon 1 and exon 2 probes. The yellow arrow shows a single probe hybridization (exon 1). Scale bar represents $10 \mu \mathrm{M}$.

invaded D. yakuba, another species from the melanogaster group.

In conclusion, we provide the first report on the presence of the $P$-element in South American populations of $D$. simulans. This element has not been previously detected in samples collected between 2002 and 2009, but was found in specimens from five localities collected between 2018 and 2019. It is worth mentioning that $P$-elements were not detected in flies collected in Ubatuba in 2004 (Hill et al., 2016), but in our study all sampled individuals from this locality presented the $P$-element, just 14 years later. Altogether, our results showed that this invasion is at an advanced stage and, together with other recent studies, confirms the trend of a remarkable rapid spread of $P$-elements across worldwide populations of $D$. simulans.

\section{Acknowledgments}

We are thankful to Pedro Heringer for $D$. simulans identification, Lucas Assunção for his help with the map figure and Erick Junqueira for his help with collecting Drosophila. This research was funded by Conselho Nacional de Desenvolvimento Científico e Tecnológico (CNPq) (grant number 404620/2016-7 and fellowship 308386/2018-3 to G.C.S.K and fellowship 310433/2018-5 to M.S.; fellowship 116558/2019-9 to A.M.L.N) and partially by the Coordenação de Aperfeiçoamento de Pessoal de Nível Superior - Brasil (CAPES) - Finance Code 001).

\section{Conflict of Interest}

The authors declare that there is no conflict of interest that could be perceived as prejudicial to the impartiality of the reported research.

\section{Authors Contributions}

AMLN and GCSK conceived the study, AMLN and BSMLS conducted the experiments, AMLN analysed the data, AMLN and GCSK wrote the manuscript, BSMLS and MS revised the manuscript, all authors read and approved the final version.

\section{References}

Anxolabéhère D, Kidwell MG and Periquet G (1998) Molecular characteristics of diverse populations are consistent with the hypothesis of a recent invasion of Drosophila melanogaster by mobile P elements. Mol Biol Evol 3:252-269.

Bartolomé C, Bello X and Maside X (2009) Widespread evidence for horizontal transfer of transposable elements across Drosophila genomes. Genome Biol 10:R22.

Bourque G, Burns KH, Gehring M, Gorbunova V, Seluanov A, Hammell M, Imbeault M, Izsvák Z, Levin HL, Macfarlan TS et al. (2018) Ten things you should know about transposable elements. Genome Biol 19:199.

Castro JP and Carareto CM (2004) Drosophila melanogaster P transposable elements: mechanisms of transposition and regulation. Genetica 21:107-118.

Clark JB, Maddison WP and Kidwell MG (1994) Phylogenetic analysis supports horizontal transfer of $\mathrm{P}$ transposable elements. Mol Biol Evol 11:40-50.

Dias GB, Svartman M, Delprat A, Ruiz A and Kuhn GCS (2014) Tetris is a foldback transposon that provided the building blocks for an emerging satellite DNA of Drosophila virilis. Genome Biol Evol 6:1302-1313.

Hill T, Schlötterer C and Betancourt AJ (2016) Hybrid dysgenesis in Drosophila simulans associated with a rapid invasion of the P-element. PLoS Genet 3:e1005920.

Karpen GH and Spradling AC (1992) Analysis of subtelomeric heterochromatin in the Drosophila minichromosome Dp1 187 by single P element insertional mutagenesis. Genetics 132:737-753. 
Kidwell MG and Lisch DR (2001) Perspective: transposable elements, parasitic DNA, and genome evolution. Evolution 55:1-24.

Kofler R, Hill T, Nolte V, Betancourt AJ and Schlötterer C (2015) The recent invasion of natural Drosophila simulans populations by the P-element. Proc Natl Acad Sci U S A 21:66596663.

Kofler R, Senti KA, Nolte V, Tobler R and Schlötterer C (2018) Molecular dissection of a natural transposable element invasion. Genome Res 6:824-835.

Lee CC, Beall EL and Rio DC (1998) DNA binding by the KP repressor protein inhibits P-element transposase activity in vitro. EMBO J 14:4166-4174.

Loreto EL, Carareto CM and Capy P (2008) Revisiting horizontal transfer of transposable elements in Drosophila. Heredity 6:545-554.

Rio DC (1991) Regulation of Drosophila P element transposition. Trends Genet 7:282-287.

Sánchez-Gracia A, Maside X and Charlesworth B (2005) High rate of horizontal transfer of transposable elements in Drosophila. Trends Genet 21:200-203.

Schaack S, Gilbert C and Feschotte C (2010) Promiscuous DNA: horizontal transfer of transposable elements and why it matters for eukaryotic evolution. Trends Ecol Evol 9:537-46.

Serrato-Capuchina A, Zhang S, Martin W, Peede D, Earley E and Matute DR (2018) Recent invasion of P transposable element into Drosophila yakuba. bioRxiv:453829.

Silva JC and Kidwell MG (2000) Horizontal transfer and selection in the evolution of P elements. Mol Biol Evol 17:1542-1557.

Tamura K, Subramanian S and Kumar S (2004) Temporal patterns of fruit fly (Drosophila) evolution revealed by mutation clocks. Mol Biol Evol 21:36-44.
Yoshitake Y, Inomata N, Sano M, Kato Y and Itoh M (2018) The $\mathrm{P}$ element invaded rapidly and caused hybrid dysgenesis in natural populations of Drosophila simulans in Japan. Ecol Evol 19:9590-9599.

\section{Internet Resources}

Nascimento A, Silva BSML, Kuhn GCS and Svartman M (2020) Chromatogram Files - P-element invasion in Brazilian $D$. simulans.

Figshare, https://doi.org/10.6084/m9.figshare.11857446 (accessed14 February 2020).

\section{Supplementary material}

The following online material is available for this article: Figure S1 - Agarose gel (1.5\%) showing the PCR products with oligos (in red) designed to amplify a region of the Cid gene from $D$. simulans but not from $D$. melamogaster, as shown.

Figure S2 - Agarose gel (1.5\%) showing the PCR products from $D$. simulans using oligos designed to amplify P-element exons 0, 1, 2 and 3, as described by Hill et al. (2016).

Figure S3 - Agarose gel (1.5\%) showing the PCR products from several species captured with $D$. simulans at the bait, using oligos designed to amplify P-element exons, 1, 2 and 3, as described by Hill et al. (2016).

Associate Editor: Louis Klaczko

License information: This is an open-access article distributed under the terms of the Creative Commons Attribution License (type CC-BY), which permits unrestricted use, distribution and reproduction in any medium, provided the original article is properly cited. 